\title{
An Active Frequency Selective Surface Design Having Four Different Switchable Frequency Characteristics
}

\author{
Bora DÖKEN, Mesut KARTAL \\ Dept. of Electronics and Communications Engineering, Istanbul Technical University, Istanbul, Turkey \\ dokenb@itu.edu.tr, kartalme@itu.edu.tr
}

Submitted October 12, 2018 / Accepted February 26, 2019

\begin{abstract}
Mutual interference between indoor adjacent wireless networks is becoming an important issue due to reducing the communication speed significantly. Controlling the isolation of wireless networks by using frequency selective surfaces (FSSs) can be an efficient solution for such interference problems. Therefore, a switchable bandstop FSS design is presented in this work for unlicensed 2.4 and $5.8 \mathrm{GHz}$ ISM (Industrial, Scientific and Medical) bands. In order to achieve desired frequency switch performance, a new theory is proposed in this paper by using PIN diodes. According to the proposed theory, PIN diodes should be placed in positions where high current densities are observed or where charges are collected. By using the proposed theory, a new FSS geometry design was carried out to control the targeted ISM bands. According to our best knowledge, there is no similar FSS work in the literature with four different frequency responses for 2.4 and $5.8 \mathrm{GHz}$ ISM bands.
\end{abstract}

\section{Keywords}

Frequency selective surface (FSS), periodic structures, wireless communication, wireless local area network (WLAN), indoor propagation, interference, active FSS

\section{Introduction}

Nowadays, unlicensed 2.4 and $5.8 \mathrm{GHz}$ ISM (Industrial, Scientific and Medical) bands are being used intensively in indoor environment. These frequency bands are especially used by WLAN (wireless local area networks) technology and the interference between adjacent networks reduces communication performance. Therefore, reducing interference in wireless networks is becoming increasingly important with the continuous deployment of larger and more sophisticated wireless networks. Isolating the wireless networks by using band-stop surfaces is an effective solution for interference problem. Band-stop filter characteristic of such surfaces can be achieved by using conducting periodic patch arrays [1], [2]. Conducting arrays are being utilized in many fields such as antenna performances improvement, multiband antennas, absorbers, band-gap filters and interference reducing in indoor environment. Such periodic conducting arrays are called as frequency selective surfaces (FSS) [1].

The intensive usage of the frequency spectrum and the usage of different frequency bands by the same wireless applications guide FSS designs to have more than one stop band. Multiband frequency response is achieved by using multiple resonators in unit cell either in nested, in hybrid or in a layered form [3-5]. In addition, today's communications technologies demand switchable [6-11] and tunable FSSs [12-18] of which their frequency selective characters can be controlled. These surfaces are called as active FSSs in literature. Researches are being done in order to control frequency response of these active FSSs. Several methods are reported in literature such as using ferrite or silicon substrates [19-24], lumped electronic elements or mechanical controls [25], [26], in FSSs. The inclusion of lumped elements within each unit cell allows controlling the frequency response by changing the applied voltage. PIN and varactor diodes are mostly used for this purpose $[6,7,9$, 11, 27-29].

In this work, an active FSS design with four different band-stop characteristics for 2.4 and $5.8 \mathrm{GHz}$ ISM bands is proposed. The proposed design consists of two layers for controlling the each of two different ISM bands. PIN diodes are integrated into the FSS in order to shift (switching feature) the stop-band resonance frequency to the out of ISM bands. Therefore, transmission or rejection of the incoming wave are being controlled depending on the applied voltage to the PIN diodes.

Separate biasing circuits are being used in order to apply bias voltage to PIN diodes. However, these circuits can limit the performance of FSSs by interfering with the incoming signal. Therefore, in this work, FSS geometry itself and the inserted lumped inductances are used as biasing circuits for PIN diodes in order to limit the unfavorable effects of biasing circuit.

Equivalent circuit (EC) models of FSS structures are efficiently used at the design and optimization stages to obtain the information on how each parameter of the periodic element works [30]. Simulations and optimizations of the proposed geometry are executed by using Ansoft HFSS v.18 software. 
According to our best knowledge, there is no similar FSS work in the literature with four different frequency responses for 2.4 and $5.8 \mathrm{GHz}$ ISM bands. Achieved results show that $20 \mathrm{~dB}$ attenuation is obtained in the ISM frequency bands where FSS is supposed to stop the incoming wave depending on the applied bias status.

Low attenuation levels are desired when the FSS is supposed to pass the incoming electromagnetic wave. Therefore, switching performance emerges an important issue. In this work, a new technique is proposed to obtain maximum switching performance in active FSS designs with PIN diodes. In order to achieve maximum switching performance, proposed technique gives information about choosing proper FSS geometries and determining proper PIN diode locations. In this work, $5 \mathrm{~dB}$ and $7.5 \mathrm{~dB}$ attenuations are achieved at 2.4 and $5.8 \mathrm{GHz}$ ISM bands, respectively, where the proposed active FSS is supposed to pass the incoming wave. Switching performance has not been adequately discussed in the literature. $3 \mathrm{~dB}\left(\mathrm{~S}_{21}\right)$ and less attenuations can be achieved in the transmission mode of FSS designs that control a single frequency band [6], [29]. However, the proposed design controls two frequency bands and its two-layer structure at least doubles the insertion loss of the FSS. One of the stop-bands can also substantially reduce the switching performance of the other stop-band. There are no similar studies in the literature to compare. Therefore, achieved attenuation levels and the effect of the electrical properties of the PIN diodes on the switching performance of the proposed FSS are also discussed in this work.

In this paper, design process, simulation and measurement results are presented in Sec. 2 and the results are discussed in Sec. 3.

\section{Design and Measurement}

An active FSS design with different band-stop characteristics in 2.4 and $5.8 \mathrm{GHz}$ ISM bands is presented in this work. PIN diodes are integrated into the FSS in order switch the behavior of FSS between transmission and rejection of the incoming wave by applying bias voltages. At the first design stage, "Circular Loop" geometry (Fig. 1(a)) is chosen as initial FSS geometry due to its polarization stability performance and simple structure. Four PIN diodes are then integrated into the FSS geometry with 90 degrees intervals in order to achieve desired frequency shifting (switching) performance. Subsequently, the conducting path widths at the connection points of PIN diodes are increased to improve the switching performance of FSS and to make the soldering of PIN diodes easier (Fig. 1(b)).

PIN diodes behave as a capacitor when they are reverse biased (OFF mode). Otherwise, they behave as a small valued variable resistance when they are forward biased (ON mode). This resistance value depends on the amount of current flowing through the diode. The brand, number and locations of the PIN diodes in unit cell mainly affect the performance of FSS. NXP BAP 50-02 PIN diode

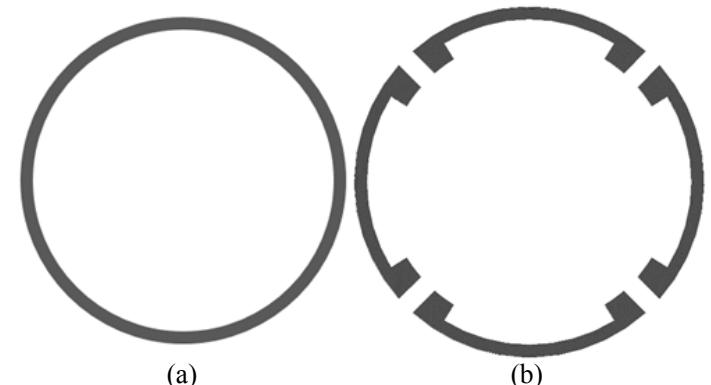

(a)

(b)

Fig. 1. (a) Circular loop geometry. (b) Improved geometry.

is chosen in the design due to its reasonable price and a low equivalent capacitance value.

PIN diodes are modelled by using their equivalent lumped element circuit in simulations. However, when RF signal is applied onto PIN diodes, charge carriers move into the intrinsic layer of PIN diode (i-layer), thereby, reducing the diode's impedance or capacitance depending on the operation mode of diode. Therefore, reversed bias equivalent capacitance of PIN diodes depends on frequency and amplitude of the incoming RF signal, PIN diode's "i-layer" width and applied bias voltage [31].

In this work, reverse bias $(0 \mathrm{~V})$ equivalent capacitance of BAP 50-02 is calculated experimentally. Prototype of the FSS is measured under $0 \mathrm{dBm}$ RF exposure condition. According to the comparison of simulation and measurement results, BAP 50-02 PIN diode is modeled with $0.2 \mathrm{pF}$ (instead of $0.4 \mathrm{pF}$ in the datasheet) capacitance and $3 \Omega$ $(10 \mathrm{~mA})$ resistance at the OFF and ON modes, respectively.

Biasing circuits have to be considered to feed the PIN diodes in the unit cell of FSS. Separate biasing circuits for PIN diodes interfere with the incoming wave. Therefore, conducting paths in the unit cell geometries are used as biasing circuit in order to bias PIN diodes. For this purpose, inductances $(22 \mathrm{nH})$ are used to conduct biasing DC voltage between unit cells (Fig. 2) and to separate the unit cell geometries in the frequency band under consideration. Biasing voltage value is determined by the number of forward-biased PIN diodes. According to study in [32], periodic structures must have minimum of $15 \times 15$ elements to be able to exhibit frequency behavior similar to a FSS with infinite number of elements. Therefore, in order to use lower biasing voltage values without effecting the frequency response of the FSS, FSS must be divided into subsections with minimum $15 \times 15$ elements for proper ground and biasing connections, as shown in Fig. 2.

According to equivalent circuit theory, equivalent inductances of the conducting paths are related with the length $(d)$ and the width $(w)$ of the conducting paths $L \propto d / w$. Equivalent capacitances are related with the gap $(g)$ and the length of the gap between conducting paths of the structure $C \propto$ length of $g a p / g$. In this work, equivalent circuit models (Fig. 2(b) and (c)) are presented to acquire PIN diodes', inductors' and unit cell geometry's influence on the frequency response of FSS. In these models, $C_{\mathrm{d}}$ and 


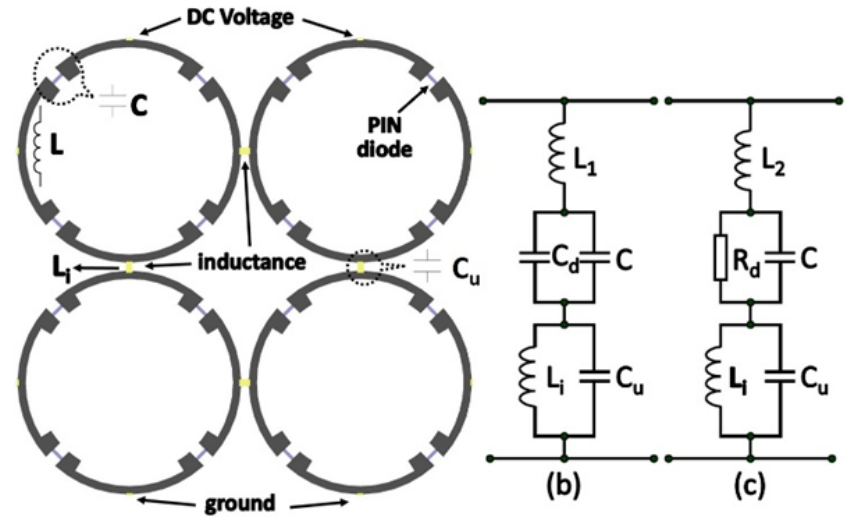

Fig. 2. (a) FSS structure, Equivalent circuit model: (b) PIN diodes reverse biased (OFF). (c) PIN diodes forward biased (ON)circuit.

$R_{\mathrm{d}}$ denotes the equivalent inductance and resistance values of PIN diodes respectively. $L_{\mathrm{i}}$ denotes the inductance of the integrated inductors. $L_{1}, L_{2}$ denote inductances of the conducting paths and $C, C_{\mathrm{u}}$ denote the equivalent capacitances between the conducting paths, as shown in Fig. 2.

When the impedance $(Z)$ of the FSS approaches to zero, the surface acts as a reflector for the incoming wave. The ON and OFF modes impedances of the FSS $\left(Z_{\mathrm{OFF}}\right.$ and $\left.Z_{\mathrm{ON}}\right)$ and the resonance frequencies $\left(f_{\mathrm{rOFF}}\right.$ and $\left.f_{\mathrm{rON}}\right)$ are derived from the given equivalent circuit model as in $(1-4)$, respectively. It is obvious that equivalent capacitance $C$ is negligible in comparison with equivalent capacitance $C_{\mathrm{d}}$ of PIN diode in OFF mode equations. Equivalent capacitance $C$ is also neglected in ON mode equations (2), (3) due to showing rather high impedance values in the desired frequency band in comparison with the equivalent resistance of PIN diode $R_{\mathrm{d}}$. The term $w^{4} L_{1} L_{\mathrm{i}} C_{\mathrm{u}} C_{\mathrm{d}}$ in (1) is also neglected in resonant frequency equation (2) due to having rather lower values comparing with the other terms.

$$
\begin{gathered}
Z_{\mathrm{OFF}}=\frac{1-\left(w^{2} L_{\mathrm{i}} C_{\mathrm{u}}\right)-\left(w^{2} C_{\mathrm{d}}\left(L_{1}+L_{\mathrm{i}}\right)\right)+\left(w^{4} L_{1} L_{\mathrm{i}} C_{\mathrm{u}} C_{\mathrm{d}}\right)}{\mathrm{j} w C_{\mathrm{d}}\left(1-w^{2} L_{\mathrm{i}} C_{\mathrm{u}}\right)}, \\
f_{\mathrm{rOFF}}=\frac{1}{2 \pi \sqrt{L_{\mathrm{i}} C_{\mathrm{u}}+C_{\mathrm{d}}\left(L_{1}+L_{\mathrm{i}}\right)}}, \\
Z_{\mathrm{ON}}=R_{\mathrm{d}}+\frac{\mathrm{j} w\left(L_{\mathrm{i}}+L_{2}\right)-\mathrm{j} w^{3} L_{\mathrm{i}} L_{2} C_{\mathrm{u}}}{\left(1-w^{2} L_{\mathrm{i}} C_{\mathrm{u}}\right)}, \\
f_{\mathrm{rON}}=\frac{\sqrt{L_{\mathrm{i}}+L_{2}}}{2 \pi \sqrt{L_{\mathrm{i}} L_{2} C_{\mathrm{u}}}} .
\end{gathered}
$$

At the design stage, brand and models of PIN diode and inductance elements are determined first in accordance with their electrical properties, price and availability. The gap distance between unit cell geometries and the gap distance between unit conducting paths are then determined by the package model of integrated inductors and PIN diodes, respectively. The length and width of conducting paths are two main remaining parameters at the optimization stage. These two parameters of the FSS are optimized for $2.45 \mathrm{GHz}$ resonance frequency in accordance with the information obtained from equivalent circuit model, by using the parametric analysis feature of Ansoft HFSS software.

On the other hand, reversed biased PIN diodes behave as a capacitor and thereby, their positions on FSS geometry are very important for switching performance. PIN diodes should be placed in positions where high current densities are observed or where charges are collected. Therefore, a simple FSS geometry (circular loop) consisting of only one conducting path is chosen in the design stage. Besides, according to the proposed technique described above, the path widths to which the diodes are connected are optimized to improve the switching performance of FSS. Obtained results show that the difference between $\mathrm{ON}$ and OFF modes resonance frequencies is increased $11 \%$ from $0.86 \mathrm{GHz}$ to $0.96 \mathrm{GHz}$ in comparison with "Circular loop" geometry as shown in Tab. 1.

At the next stage, the proposed periodic structure (Fig. 2(a)) is duplicated on a new layer (bottom layer) to obtain dual band-stop behavior as shown in Fig. 3. Upper (top) and bottom layers of the new FSS control 2.4 and $5.8 \mathrm{GHz}$ ISM band transmissions, respectively. Bottom layer geometry that controls the $5.8 \mathrm{GHz}$ ISM band is smaller in size than the upper layer geometry. Due to their smaller dimensions in unit cell, bottom layer geometries are connected to each other via conducting paths (Fig. 3(c): " $a$ " parameter) as shown in Fig. 3(c). Inductors $(22 \mathrm{nH})$ are then integrated into the conducting paths to separate the unit cells electrically in the desired frequency band.

$5.8 \mathrm{GHz}$ ISM band is less stable than the $2.4 \mathrm{GHz}$ ISM band for oblique incidence angles due to the distance between adjacent bottom geometries is larger than the top layer geometries. Besides, separated conducting paths (Fig. 3(c): " $a$ " parameter) of the bottom layer interferes with the incoming RF signal. Therefore, in order to decrease dimension differences between top and bottom layer, stop-band resonance frequencies $(2.4$ and $5.8 \mathrm{GHz})$ are shifted to the out of ISM bands between 2.4 and $5.8 \mathrm{GHz}$ frequencies. The stop-band resonant frequencies are shifted to lower frequencies when the PIN diodes are switched from OFF mode to ON mode. Therefore, the top

\begin{tabular}{|c|c|c|c|}
\hline & $\begin{array}{c}\text { Diodes ON } \\
\text { Resonance }\end{array}$ & $\begin{array}{c}\text { Diodes OFF } \\
\text { Resonance }\end{array}$ & Switching \\
\hline Circular loop & $2.64 \mathrm{GHz}$ & $3.5 \mathrm{GHz}$ & $0.86 \mathrm{GHz}$ \\
\hline Improved & $2.58 \mathrm{GHz}$ & $3.54 \mathrm{GHz}$ & $0.96 \mathrm{GHz}$ \\
\hline
\end{tabular}

Tab. 1. Switching performance of FSS geometries in Fig. 1.

\begin{tabular}{|l|l|l|l|}
\hline Top layer & Bottom layer & Status & Bias Modes \\
\hline Diodes ON & Diodes ON & $\begin{array}{l}2.4 \mathrm{GHz} \text { stop, } \\
5.8 \mathrm{GHz} \text { allow }\end{array}$ & F1 \\
\hline Diodes ON & Diodes OFF & $\begin{array}{l}2.4 \mathrm{GHz} \text { and } \\
5.8 \mathrm{GHz} \text { stop }\end{array}$ & F2 \\
\hline Diodes OFF & Diodes ON & $\begin{array}{l}2.4 \mathrm{GHz} \text { and } \\
5.8 \mathrm{GHz} \text { allow }\end{array}$ & F3 \\
\hline Diodes OFF & Diodes OFF & $\begin{array}{l}2.4 \mathrm{GHz} \text { allow, } \\
5.8 \mathrm{GHz} \text { stop }\end{array}$ & F4 \\
\hline
\end{tabular}

Tab. 2. Different bias modes of FSS (stop: no transmission through the surface; allow: transmission through the surface). 
layer geometry is optimized to stop $2.4 \mathrm{GHz}$ ISM band when PIN diodes are forward biased (ON). Similarly, bottom layer is optimized to stop $5.8 \mathrm{GHz}$ incoming signals when the diodes are reversed biased (OFF). Four different bias modes are denoted in Tab. 2 .

Dual-layer FSS (Fig. 3) is optimized by using parametric analysis feature of Ansoft HFSS software similar to the optimization of single-layer structure. The following dimensions are obtained at the end of the optimization process: $\mathrm{h}=1.6 \mathrm{~mm}$ (thickness of the FR4 substrate), $\mathrm{g}=0.65 \mathrm{~mm}$ (gap between the periodic elements) and the other values in $(\mathrm{mm})$ are $\mathrm{p}=27, \mathrm{t} 1=11.4$, $\mathrm{t} 2=13.4, \mathrm{t} 3=12.5, \mathrm{t} 4=0.45, \mathrm{t} 5=0.7, \mathrm{t} 6=63.37^{\circ}, \mathrm{t} 7=10.04^{\circ}$, $\mathrm{t} 8=1.3, \mathrm{t} 9=0.3, \mathrm{~b} 1=6.8, \mathrm{~b} 2=8.8, \mathrm{~b} 3=7.7, \mathrm{~b} 4=6.8, \mathrm{~b} 5=0.7$, $\mathrm{b} 6=0.45, \mathrm{~b} 7=50.37^{\circ}, \mathrm{b} 8=14.32^{\circ}, \mathrm{b} 9=1.3$ and $\mathrm{b} 10=0.3$.

The prototype of FSS (Fig. 4) structure was manufactured on $270 \mathrm{~mm} \times 270 \mathrm{~mm} \times 1.6 \mathrm{~mm}$ FR4 substrate with dielectric constant of 4.5 for laboratory measurements.

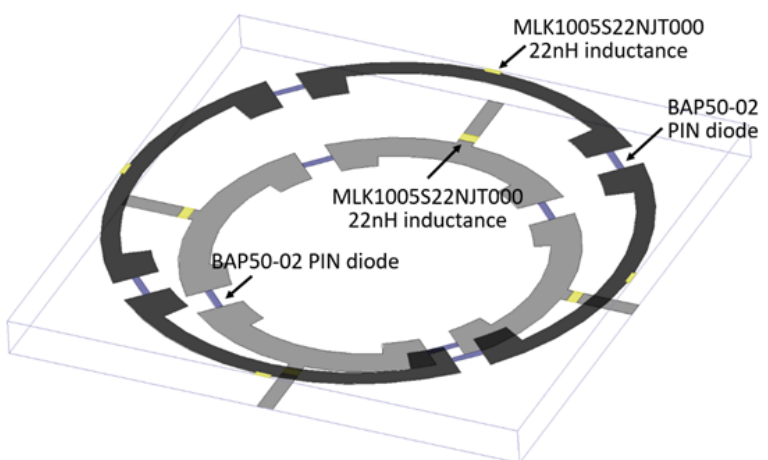

(a)

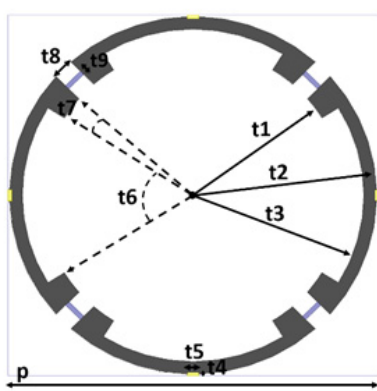

(b)

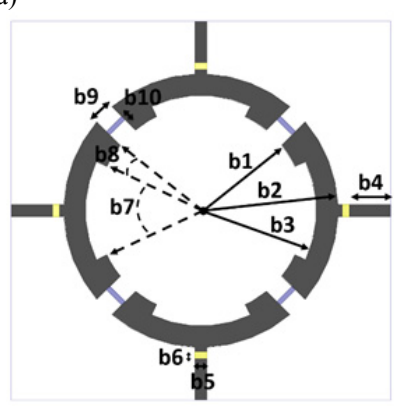

(c)
Fig. 3. FSS geometry (a) Trimetric view. (b) Top layer. (c) Bottom layer.

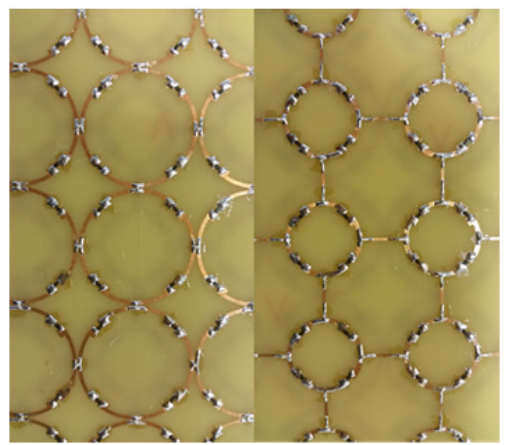

(a) Top layer

(b) Bottom layer

Fig. 4. Prototype.
The measurements for the fabricated prototype were performed by Agilent N5230A model network analyzer and with two Schwarzbeck Mess BBHA 9120D-model horn antennas. A thru calibration was performed to calibrate the network analyzer within the frequency band of $1.8-6.3 \mathrm{GHz}$ in the absence of the FSS. The transmitting and receiving antennas were at the same height and located on the line-of-sight direction.

Figures 5-12 show the simulation (s) and measurement $(\mathrm{m})$ results for different bias configurations (Tab. 2) for both TE and TM polarizations, respectively. Achieved simulation results show that minimum $20 \mathrm{~dB}$ attenuation is obtained in frequency bands where FSS is supposed to stop the incoming wave depending on its bias mode. Besides, achieved maximum attenuations are obtained as $5 \mathrm{~dB}$ and $7.5 \mathrm{~dB}$ for 2.4 and $5.8 \mathrm{GHz}$ ISM bands, respectively, where FSS is supposed to pass the incoming wave. Attenuation levels of the "allow" state are directly affected by the bandwidth of the out-of-band resonances. According to filter circuit theory, bandwidth of the stop-band increases with the increase of equivalent circuit capacitance at LC series circuits. The proof of this theory can be clearly observed on the measurement and simulation results. Bandwidths of the reverse biased modes (F4) mode are larger than the forward bias modes (F1 mode). Therefore, the use of PIN diodes with smaller equivalent capacitance values can further reduce attenuation values in the transmission mode of the design.

It is also observed that the frequency stability of the proposed design is lower at high frequencies $(5.8 \mathrm{GHz}$ frequency band) as expected, because the distance between adjacent bottom layer geometries (controls $5.8 \mathrm{GHz}$ band) is larger than the top layer geometries. Frequency shift in the $5.8 \mathrm{GHz}$ frequency band also affects the $2.4 \mathrm{GHz}$ band negatively because of the mutual interference between each resonant geometry.

Frequency shifts are observed in between measurement and simulation results, especially in the $5.8 \mathrm{GHz}$ frequency band. As the dimensions of the FSS geometries become smaller, fabrication errors become more important and lead to further shifts in the frequency response of the surfaces. Therefore, high frequency behavior error of lumped elements (PIN diodes and inductors), the coarse soldering processes, placing errors of the lumped elements and experimental equivalent capacitance determination errors of the of PIN diodes become more important on frequency shifts. Furthermore, finite size of the prototype allows diffraction at the edges especially at the low frequency signals. Therefore, achieved measurement attenuations in $2.4 \mathrm{GHz}$ ISM band are generally less than simulation results, unlike $5.8 \mathrm{GHz}$ ISM band.

Figure 3(b) and 3(c) geometries are simulated separately for the PIN diodes ON and OFF conditions in order to see the mutual coupling effect between layers (Fig. 13). According to the comparison of simulation results (Fig. 5-12) with the results in Fig. 13, it is observed that mutual coupling effect slightly decreases the resonance frequencies. 


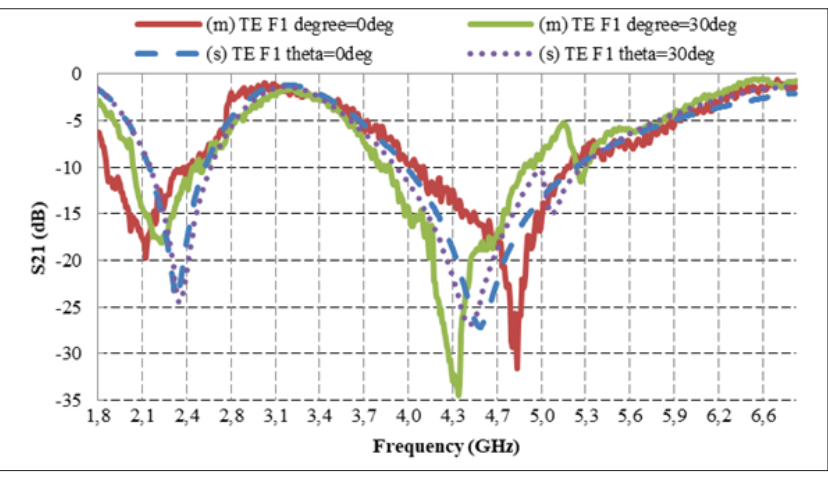

Fig. 5. $\mathrm{S}_{21}$ frequency curves for $\mathrm{F} 1$ bias configurations at TE polarization.

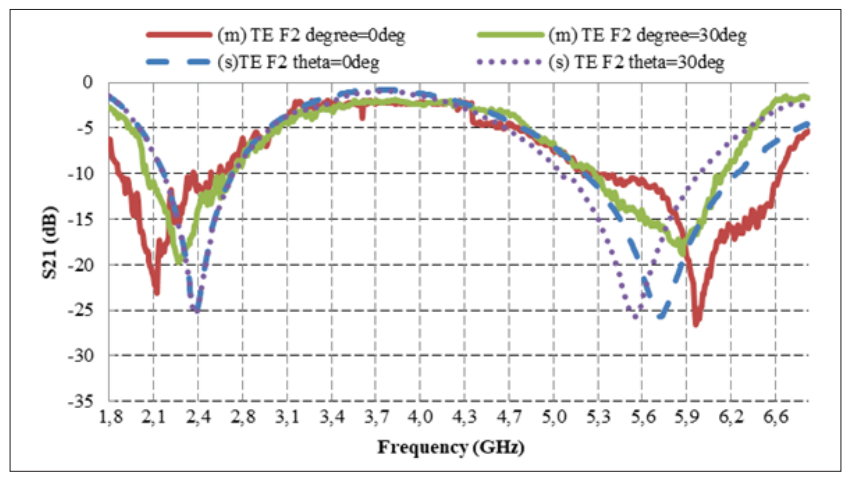

Fig. 6. $\mathrm{S}_{21}$ frequency curves for $\mathrm{F} 2$ bias configurations at TE polarization.

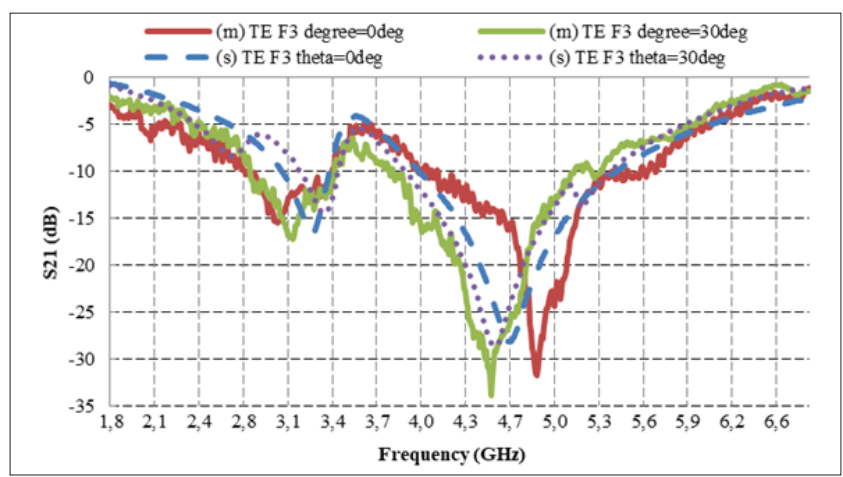

Fig. 7. $\mathrm{S}_{21}$ frequency curves for $\mathrm{F} 3$ bias configurations at $\mathrm{TE}$ polarization.

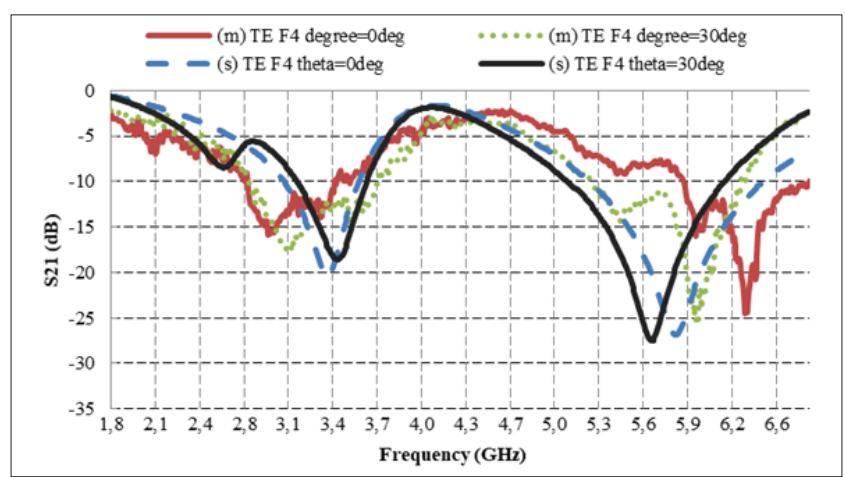

Fig. 8. $\mathrm{S}_{21}$ frequency curves for $\mathrm{F} 4$ bias configurations at TE polarization

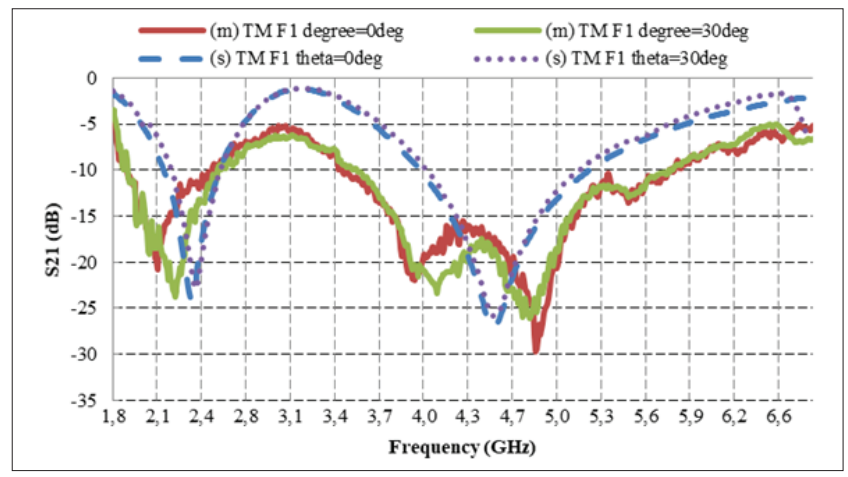

Fig. 9. $S_{21}$ frequency curves for F1 bias configurations at TM polarization.

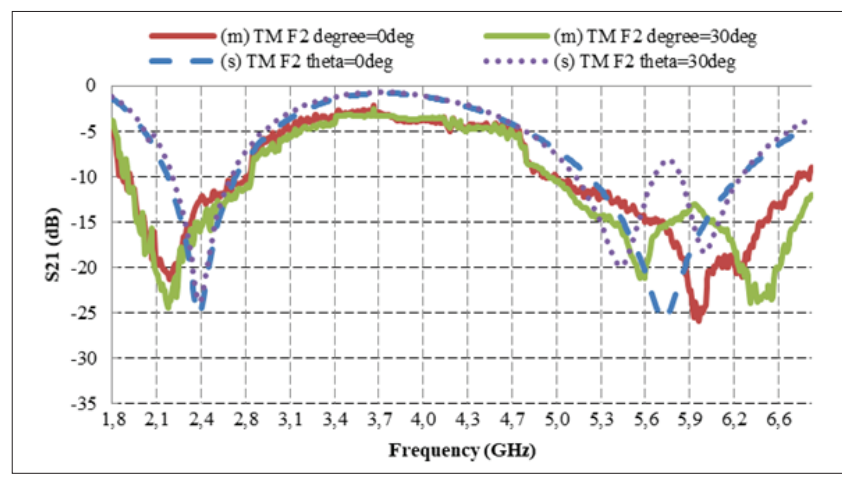

Fig. 10. $S_{21}$ frequency curves for $\mathrm{F} 2$ bias configurations at TM polarization.

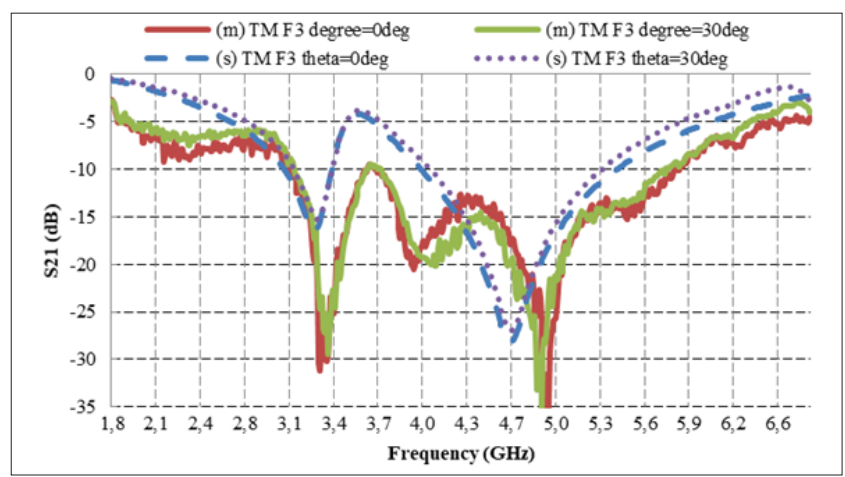

Fig. 11. $\mathrm{S}_{21}$ frequency curves for $\mathrm{F} 3$ bias configurations at $\mathrm{TM}$ polarization.

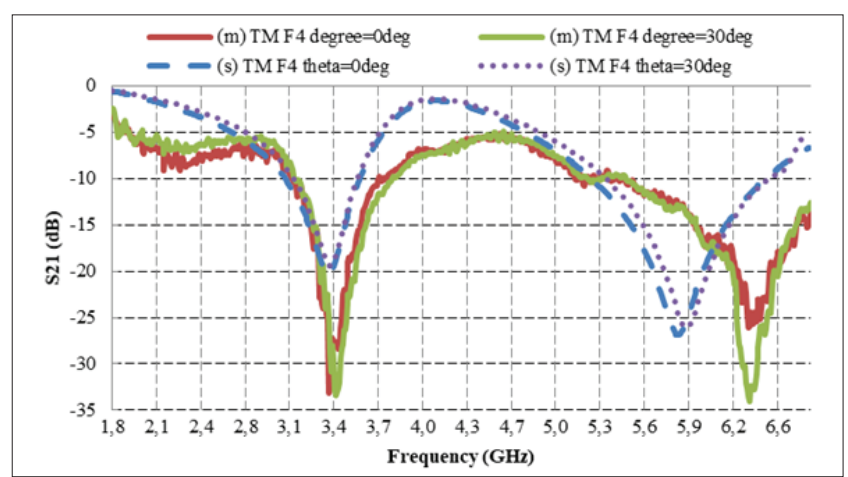

Fig. 12. (a) $S_{21}$ frequency curves for $F 4$ bias configurations at TM polarization. 


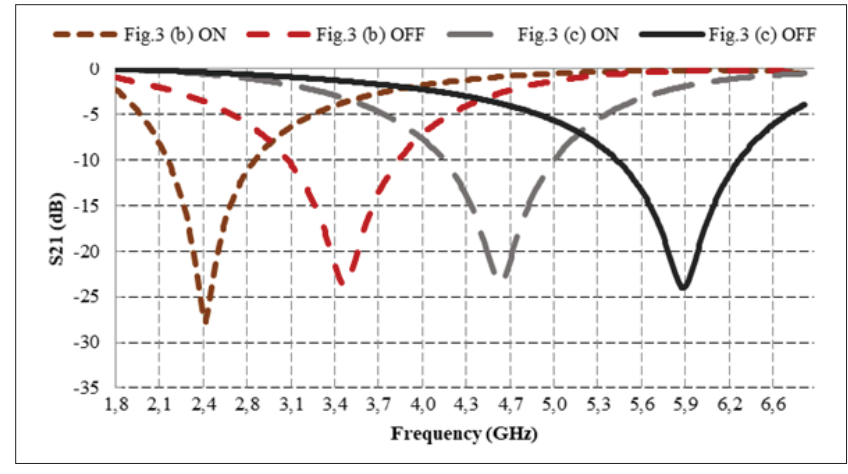

Fig. 13. $\mathrm{S}_{21}$ frequency curves of Fig. 3(b) and Fig. 3(c) geometries for PIN diodes forward $(\mathrm{ON})$ and reverse biased (OFF) conditions at TE polarization.

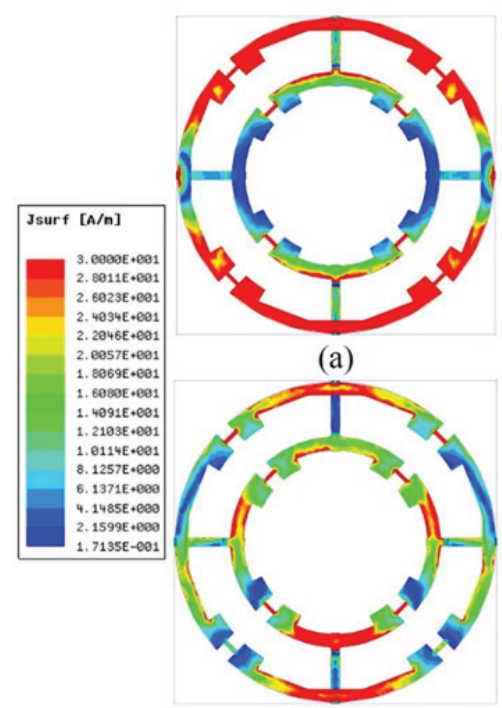

(c)

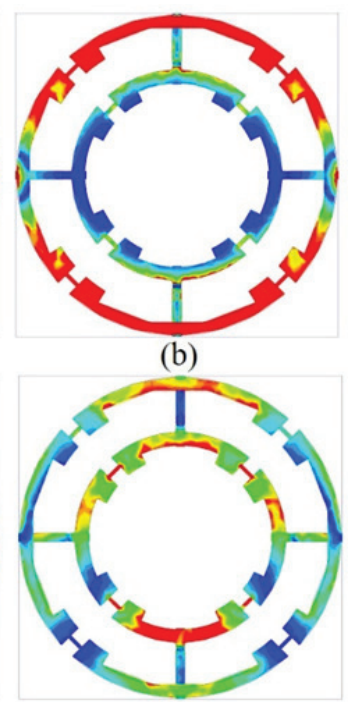

(d)
Fig. 14. Current distribution diagrams: (a) F1 mode, $2.4 \mathrm{GHz}$, (b) F2 mode, $2.4 \mathrm{GHz}$, (c) F2 mode, $5.8 \mathrm{GHz}$, (d) F4 mode, $5.8 \mathrm{GHz}$.

According to the proposed theory in this paper, PIN diodes should be placed in positions where high current densities are observed. Therefore, current distribution diagrams of FSS geometries when they resonate are given in Fig. 14 for $2.4 \mathrm{GHz}$ and $5.8 \mathrm{GHz}$ frequencies. It is observed that inner and outer geometries control $5.8 \mathrm{GHz}$ and $2.4 \mathrm{GHz}$ frequency bands, respectively. As expected, at resonance frequencies, high current densities are observed on PIN diodes of the geometries, which resonate.

\section{Conclusion}

A band stop FSS geometry is designed for WLAN security and interference mitigating in the 2.4 and $5.8 \mathrm{GHz}$ ISM bands. Proposed FSS have four switchable frequency characteristics depending on the applied bias voltage. Achieved results show that $20 \mathrm{~dB}$ attenuation is obtained in frequency bands where FSS is supposed to stop the incoming wave depending on the applied bias status. Desired switching performance is achieved by applying a technique, which helps finding the proper locations of the PIN diodes in FSS geometries. The obtained thickness of the structure is only $1.6 \mathrm{~mm}$, which also gives the possibility of using this design as a structural surface material for blocking the ISM signals.

\section{Acknowledgments}

We would like to thank TUBITAK (project no: 115E225) and Istanbul Technical University BAP foundation (project no: 37010) for funding this work.

\section{References}

[1] MUNK, B. A. Frequency Selective Surfaces - Theory and Design. New York: John Wiley and Sons. Inc., 2000

[2] WU, T. K. Frequency Selective Surface and Grid Array: Wiley Interscience Publication, 1995

[3] OMAR, A. A., SHEN, Z. Multiband high-order bandstop 3-D frequency-selective structures. IEEE Transactions on Antennas and Propagation, 2016, vol. 64, no. 6, p. 2217-2226. DOI: 10.1109/tap.2016.2546967

[4] FERREIRA, D., FERNANDES, T. R., CUIÑAS, I., et al. Dualband single-layer quarter ring frequency selective surface for WiFi applications. IET Microwaves, Antennas and Propagation, 2016, vol. 10, no. 4, p. 435-441. DOI: 10.1049/iet-map.2015.0641

[5] RAHMATI, B., HASSANI, H. R. Multiband metallic frequency selective surface with wide range of band ratio. IEEE Transactions on Antennas and Propagation, 2015, vol. 63, no. 8, p. 3747-3753. DOI: 10.1109/tap.2015.2438340

[6] DÖKEN, B., KARTAL, M. Switchable frequency selective surface design for $2.45 \mathrm{GHz}$ ISM band. In 8th International Conference on Recent Advances in Space Technologies (RAST). 2017, p. 473-476. DOI: 10.1109/RAST.2017.8002969

[7] YANG, C. C., LI, H. Y., CAO, Q. S., et al. Switchable electromagnetic shield by active frequency selective surface for LTE-2.1 GHz. Microwave and Optical Technology Letters, 2016, vol. 58, no. 3, p. 535-540. DOI: 10.1002/mop.29617

[8] ZAHRA, H., RAFIQUE, S., SHAFIQUE, M. F., et al. A switchable frequency selective surface based on a modified Jerusalem-cross unit cell. In 2015 9th European Conference on Antennas and Propagation (EuCAP). Lisbon (Portugal), 2015, p. 1-2

[9] MAHMOOD, S. M., T. A. DENIDNI, T. A. Switchable square loop frequency selective surface. Progress In Electromagnetics Research Letters, 2015, vol. 57, p. 61-64. DOI: 10.2528/PIERL15090402

[10] KONG, P., YU, X. W., ZHAO, M. Y., et al. Switchable frequency selective surfaces absorber/reflector for wideband applications. Journal of Electromagnetic Waves and Applications, 2015, vol. 29, no. 11, p. 1473-1485. DOI: 10.1080/09205071.2015.1049713

[11] FABIAN-GONGORA, H., MARTYNYUK, A. E., RODRIGUEZCUEVAS, J., et al. Active dual-band frequency selective surfaces with close band spacing based on switchable ring slots. IEEE Microwave and Wireless Components Letters, 2015, vol. 25, no. 9, p. 606-608. DOI: 10.1109/LMWC.2015.2451358

[12] LIN BAO-QIN, QU SHAO-BO, TONG CHUANG-MING, et al. Varactor-tunable frequency selective surface with an embedded bias network. Chinese Physics B, 2013, vol. 22, no. 9, p. 1-4.

[13] BAYATPUR, F., SARABANDI, K. Tuning performance of metamaterial-based frequency selective surfaces. IEEE 
Transactions on Antennas and Propagation, 2009, vol. 57, no. 2, p. 590-592. DOI: 10.1109/tap.2008.2011404

[14] BOCCIA, L., RUSSO, I., AMENDOLA, G., et al. Tunable frequency-selective surfaces for beam-steering applications. Electronics Letters, 2009, vol. 45, no. 24, p. 1213-1215. DOI: $10.1049 / \mathrm{el} .2009 .2577$

[15] BUCHWALD, W. R., HENDRICKSON, J., CLEARY, J. V., et al. Active frequency selective surfaces. In SPIE Defense, Security, and Sensing. Baltimore (Maryland, USA), 2013, SPIE Proceedings vol. 8725. DOI: $10.1117 / 12.2016081$

[16] SAFARI, M., SHAFAI, C., SHAFAI, L. X-band tunable frequency selective surface using MEMS capacitive loads. IEEE Transactions on Antennas and Propagation, 2015, vol. 63, no. 3, p. 1014-1021. DOI: 10.1109/tap.2014.2386304

[17] SANZ-IZQUIERDO, B., PARKER, E. A., ROBERTSON, J. B., et al. Tuning technique for active FSS arrays. Electronics Letters, 2009 , vol. 45 , no. 22 , p. $1107-1109$. DOI: $10.1049 /$ el.2009.2264

[18] ZHOU, H., WANG, X., QU, S., et al. Dual-polarized FSS with wide frequency tunability and simple bias network. PIERS Proceedings, Session 1P0, 2014, p. 109-112.

[19] HU, W. F., DICKIE, R., CAHILL, R., et al. Liquid crystal tunable $\mathrm{mm}$ wave frequency selective surface. IEEE Microwave and Wireless Components Letters, 2007, vol. 17, no. 9, p. 667-669. DOI: 10.1109/LMWC.2007.903455

[20] PARKER, E. A., SAVIA, S. B. Active frequency selective surfaces with ferroelectric substrates. IEE ProceedingsMicrowaves Antennas and Propagation, 2001, vol. 148, no. 2, p. 103-108. DOI: 10.1049/ip-map:20010306

[21] LIMA, A. C. D., PARKER, E. A., LANGLEY, R. J. Tunable frequency-selective surface using liquid substrates. Electronics Letters, 1994, vol. 30, no. 4, p. 281-282. DOI: 10.1049/el:19940232

[22] Chang, T., LANGley, R. J., PARKER, E. A. Frequency selective surfaces on biased ferrite substrates. Electronics Letters, 1994, vol. 30, no. 15, p. 1193-1194. DOI 10.1049/el:19940823

[23] LI, M., BEHDAD, N. Fluidically tunable frequency selective/phase shifting surfaces for high-power microwave applications. IEEE Transactions on Antennas and Propagation, 2012, vol. 60, no. 6, p. 2748-2759. DOI: 10.1109/Tap.2012.2194645

[24] LI, M., YU, B., BEHDAD, N. Liquid-tunable frequency selective surfaces. IEEE Microwave and Wireless Components Letters, 2010, vol. 20, no. 8, p. 423-425. DOI: 10.1109/Lmwc.2010.2049257

[25] MA, D., ZHANG, W. X. Mechanically tunable frequency selective surface with square-loop-slot elements. Journal of Electromagnetic Waves and Applications, 2007, vol. 21, no. 15, p. 2267-2276. DOI: 10.1163/156939307783134407
[26] FUCHI, K., T. JUNYAN, T., CROWGEY, B., et al. Origami tunable frequency selective surfaces. IEEE Antennas and Wireless Propagation Letters, 2012, vol. 11, p. 473-475. DOI: 10.1109/lawp.2012.2196489

[27] TAYLOR, P. S., PARKER, E. A., BATCHELOR, J. C. An active annular ring frequency selective surface. IEEE Transactions on Antennas and Propagation, 2011, vol. 59, no. 9, p. 3265-3271. DOI: 10.1109/Tap.2011.2161555

[28] MIAS, C., YAP, J. H. A varactor-tunable high impedance surface with a resistive-lumped-element biasing grid. IEEE Transactions on Antennas and Propagation, 2007, vol. 55, no. 7, p. 1955-1962. DOI: $10.1109 /$ Tap.2007.900228

[29] LI, H. Y., YANG, C. C., CAO, Q. S., et al. A novel active frequency selective surface with switching performance for 2.45 GHz WLAN band. Microwave and Optical Technology Letters, 2016, vol. 58 , no. 7, p. $1586-1590$. DOI: $10.1002 /$ mop. 29855

[30] KENT, F. E., DOKEN, B., KARTAL, M. A new equivalent circuit based FSS design method by using Genetic Algoritm. In 2nd International Conference on Engineering Optimization, ENGOPT 2010. Portugal, 2010

[31] CAVERLY, R. H., HILLER, G. Establishing the minimum reverse bias for a pin diode in a high-power switch. IEEE Transactions on Microwave Theory and Techniques, 1990, vol. 38, no. 12, p. $1938-1943$. DOI: $10.1109 / 22.64577$

[32] CHEN, M., WANG, S. N.., CHEN, R. S., et al. Electromagnetic analysis of electrically large and finite periodic frequency selective surfaces. In Asia-Pacific Microwave Conference APMC. 2008, p. $1-4$.

\section{About the Authors...}

Bora DÖKEN (corresponding author) received the MS degree in 2010 and Ph.D. degree in 2018 from the Istanbul Technical University. After working in various positions in the private sector, he became an instructor at the Istanbul Technical University in October 2007. He is currently involved in the areas of FSSs, periodic structures, antennas, and metamaterials.

Mesut KARTAL (M'91) received his MS degree in 1993 and Ph.D. degree in 2000. Currently, he is a Professor in the Istanbul Technical University, Department of Electronics and Communication Engineering. His research interests include remote sensing, inverse problems, RF and microwave design engineering, as well as modelling, design, simulations and analysis, and $\mathrm{CAD}$ techniques in high frequency region. 